\title{
Validity of Modified Early Obstetric Warning System (Meows) In Low Resource Setting: A Case of St. Francis Hospital Nsambya, Kampala, Uganda
}

\author{
Article by Okello B. Otuu ${ }^{1}$, Omona Kizito ${ }^{2}$, Zaake Daniel ${ }^{3}$, Sekweyama Peter $^{4}$ \\ ${ }^{1}$ Gynaecologist, Private Practice, Uganda \& Principal Investigator \\ ${ }^{2}$ Lecturer, Uganda Martyrs' University, Faculty of Health Sciences (FHS) \& Principal \\ Investigator \\ ${ }^{3,4}$ Gynaecologist, St Francis Hospital Nsambya, Kampala
}

\begin{abstract}
Introduction: Globally every day, about 830 women die due to complications of pregnancy and child birth. Of these deaths, 99\% occur in low-resource settings, and most could be prevented. Use of Modified Early Obstetric Warning System (MEOWS) would be appropriate. MEOWS is a monitoring chart intended to identify mothers at risk and initiate the right action, at right time by the appropriately skilled clinicians, at a time when treatment might make a difference to reduce maternal mortality and morbidity.

Objectives: To determine the sensitivity, specificity and predictive values of Modified Early Obstetric Warning System (MEOWS) in correctly identifying women at risk of developing obstetric morbidity in St. Francis Hospital_Nsambya between January and February, 2016

Methods: The study was a prospective cohort study conducted at St. Francis Hospital Nsambya, maternity ward, from January to February 2016. MEOWS monitoring tool was used alongside with questionnaires.

Result: 502 respondent mothers were enrolled in the study. 160patients (31.9\%) triggered and of which: $11.5 \%$ of them had obstetric morbidity which included postpartum haemorrhage-35.5\%, preeclampsia-26.3\%, suspected infection-22.4\%, third degree perineum tear-5.3\%, anaesthetic complications-4\% and prolong hospital stay-7\%. MEOWS was $81.7 \%$ sensitive (95\% CI 80-94\%), $76.3 \%$ specific (95\% CI 74-81\%), with a positive predictive value 36.3\% (95\% CI 31-44\%) and negative predictive value of $96.2 \%$ (95\% CI 94-99\%).

Conclusion: MEOWS chart is even effective for use in low resource setting, like Uganda.
\end{abstract}

Keywords: Modified Early Obstetric Warning System (MEOWS). Sensitivity. Validity. Predictive values. Maternal Morbidity. Maternal Mortality.

\section{Introduction}

\section{Background}

Globally, about 830 women die daily due to complications of pregnancy and child birth. $99 \%$ of these deaths occur in low-resource settings. The risk of a woman in a developing country dying from a maternal-related cause during her lifetime is about 33 times higher than that of a woman living in a developed country. Most of these deaths are preventable (Say et al., 2014). Melanie (2014) argued that the principles underpinning the use of MEOWS is that patients who develop serious illness will usually display abnormalities in simple physiological parameters. Further, that if these early signs are recognized and appropriate escalation and intervention is undertaken, patient outcomes will be improved.

Uganda is one of the countries that failed to achieve the millennium development goals target of reduction of maternal mortality. The Maternal Mortality Ratio (MMR) is still high in Uganda at 438 / 100,000 live births (Uganda Bureau of Statistics [UBOS] and ICF International, 2012). About 15\% of pregnant women develop complications during their pregnancy irrespective of their geographical locations. A great difference in maternal death between developing and developed countries is due to failure of the health care system to respond when complications arise (WHO, 2005). 
One target under the Sustainable Development Goal (SDG) three is to reduce the global maternal mortality ratio to less than 70 per 100,000 births. It's argued that no country should have a maternal mortality rate of more than twice the global average (United Nations [UN], 2015).

The early detection of severe illness in pregnant women remains a challenge to many clinicians involved in their care. The relative rarity of such events, combined with the normal changes in physiology associated with pregnancy and childbirth, compounds the problem. Abnormal physiology is common among hospitalised patients. However, clinical and physiological deterioration is evident for 6-8 hours before critical illness. Serious morbidity and mortality occurs after a period of slow and progressive physiological deterioration that goes unrecognized or is inadequately treated (Marc, Helen and lauren, 2013)

Despite pregnancy and labour being normal physiological events, monitoring of vital signs is an integral part of maternity care. Regular recording and interpretation of these observations aids in the recognition of any changes in a woman's condition. There are several bedside score and track-andtrigger systems designed to aid nurses/midwives in observation of vital signs. This is aimed at facilitating early recognition of a patient's deterioration (Cioffi, 2000a).

In the United Kingdom (UK), a modified early obstetric warning system (MEOWS) has been used in obstetric inpatients to track maternal physiological parameters, and to aid early recognition and treatment of clinical deterioration. This followed a recommendation by the 2003-2005 Confidential Enquiry into Maternal and Child Health report (Lewis, 2007).

MEOWS track the following parameters: Temperature, Blood pressure, Heart rate, Respiratory rate, Oxygen saturation, Conscious level and Pain scores. The values of the observations are then translated into a summary score which has a critical threshold, above which medical review and intervention is required. Using MEOWS prompts early medical evaluation and referral to the appropriate practitioner.

There also, exist, a number of challenges associated with implementing MEOWS. A study by Isaacs et al. (2014) found the following challenges; Staffing pressures preventing adequate completion of charts (35\%), Lack of support for EWS charts from midwives (22\%) and Lack of teaching/training (21\%). Other challenges were concurrent use of a standard vital signs chart/partograph (20\%), too timeconsuming (14\%), lack of support for MEOWS charts from doctors (9\%), Lack of evidence and validation of MEOWS in obstetrics (9\%), impact on the mother of frequent interruptions (8\%) and Poor correlation of charts with obstetric physiology $(7 \%)$

\section{Problem statement}

Maternal morbidity and mortality are highest around the time of delivery and early postpartum period (WHO, 2005). This is, predominantly, due to postpartum haemorrhage, preeclampsia and sepsis, which are all preventable if detected early. Early detection calls for closer monitoring of both mother and baby during this time.

Monitoring of obstetric inpatient is a problem as cited by Centre for Maternal and Child Enquiries (CMACE) report during their enquiry into maternal death in the United Kingdom (Lewis, 2007). Similar report was release by Ministry of Health (MOH) _ Uganda (MOH, 2010).

Locally the tools used do not guide the clinicians well enough on abnormal measurements. But even then, it depends on the clinicians' knowledge of an abnormal test result. A MEOWS is a tool that guides the clinician based on colour codes for an abnormal result.

MEOWS is a monitoring tool recommended by CMACE for predicting mothers at risk of developing maternal morbidity and is currently being used in UK hospitals. However, no such tool has been validated for use in low income setting and data on the use of such tools is limited.

The researchers thought of it as critical to determine the applicability of MEOWS as an essential tool in the prediction of maternal morbidity in the context of developing countries such as Uganda.

\section{Conceptual frame work}

Soon after delivery (both Spontaneous Vaginal Delivery (SVD) and Caesarean Section (C/S)), the mothers are monitored using Modified Early Obstetric Warning Score (MEOWS). The result is either a 'Triggers' or 'No triggers'. In case of no triggers, the doctor monitors the mother after 6hour. In case of triggers (1Red and 2Yellows), the doctor is called to review the mother immediately. At discharge, 
the doctor reviews the chart for evidence for any maternal morbidity or mortality. Refer to figure 1 below;

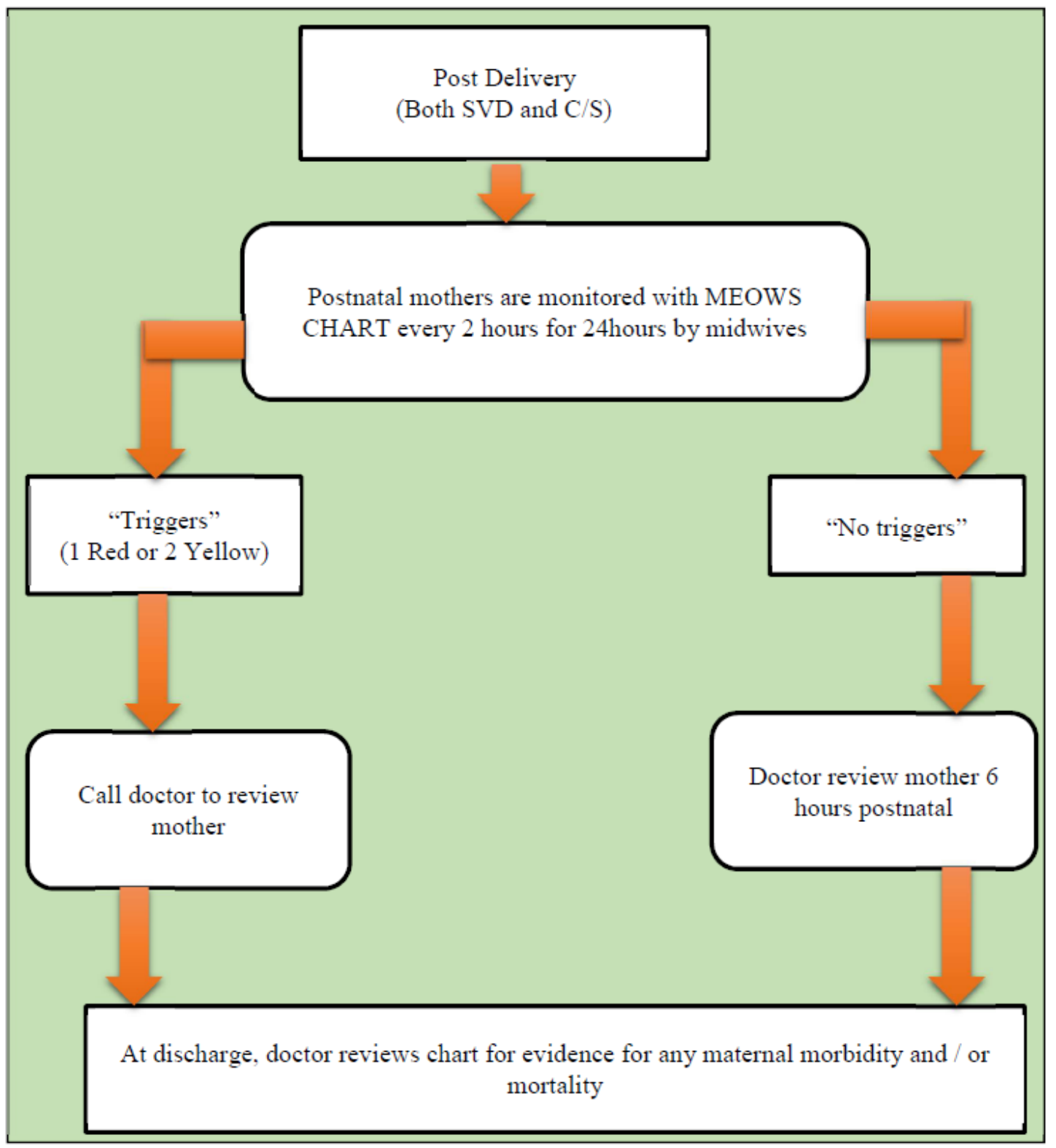

Figure1. Flow of events during application of MEOWS

\section{Objectives}

The study had both general and specific objectives as below;

\section{General objective}

The general objective was to validate the usage of MEOWS as a tool for predicting risk of developing maternal morbidity in Uganda

\section{Specific objective}

The specific objective was to determine the sensitivity, specificity and predictive values of MEOWS in correctly identifying a woman at risk of developing obstetric morbidity in St. Francis Hospital Nsambya. Between January and February, 2016. 
DOI: 10.21522/TIJCR.2014.05.01.Art006

ISSN: $2520-3096$

\section{Justification of the study}

Modified Early Obstetric Warning System (MEOWS) was introduced with the aim of improving patients monitoring. It was first used in UK hospitals as monitoring tool to correctly identify women at risk of developing obstetric morbidity. Early recognition and treatment could reduce maternal morbidity and death. However, no such tool has been validated for use in low resource setting and data on the use of such tools is limited. It is critical to determine the applicability of MEOWS as an essential tool in the prediction of Maternal Morbidity in the context of the developing country like Uganda. This information might be useful to both clinicians and policy makers in reducing maternal Morbidity and death.

\section{Research methodology}

\section{Study design}

This was prospective cohort study carried out between January and February 2016.

\section{Study setting}

The study was conducted in the obstetrics and gynaecology department of St. Francis Hospital Nsambya. The hospital is a Catholic founded Private-Not-For-Profit hospital located in the Southern part of Kampala city. It is about three kilometres from the city central business area. It is a tertiary referral hospital with a bed capacity of 361. Its designated catchment area is Makindye West Health Sub-District and it's owned by Kampala Catholic Archdiocese.

The clinical staffs working in the hospital's maternity wards include; Obstetricians / Gynaecologists, Senior House Officers on specialty training, Intern Doctors, Registered Midwives, Enrolled Midwives and Nursing Assistants

\section{Study population}

All postnatal mothers who had delivered from St. Francis Hospital Nsambya during the study period.

\section{Sampling}

Postnatal mothers who consented for the study were recruited using consecutive sampling method until the required numbers.

\section{Sample size}

Buderer's formula were applied to calculate the sample size as follows (Buderer, 1996)

Sample size estimation $(n)=\left[\frac{z_{1-\frac{\alpha}{2}}^{2} S_{N}\left(1-S_{N}\right)}{\varepsilon^{2} X \text { Prevalence }}\right]=\left[\frac{1.96^{2} \times 0.89 \times(1-0.89)}{0.05^{2} X 0.3}\right]$

$$
\begin{aligned}
& \Rightarrow n=\mathbf{5 0 1 . 4 5 6 8 5} \\
& \Rightarrow n=\mathbf{5 0 2}
\end{aligned}
$$

Where;

$\mathbf{S}_{\mathbf{N}}=$ Anticipated sensitivity of $89 \%=\mathbf{0 . 8 9}$ (Singh, McGlennan, England and Simons, 2012)

Prevalence $=$ Prevalence of disease in population, which can be obtained from previous literature $=$ $30 \%=\mathbf{0 . 3}$ (Singh, McGlennan, England and Simons, 2012)

$\boldsymbol{\varepsilon}^{2}=$ Required absolute precision on either side of the sensitivity $=5 \%=\mathbf{0 . 0 5}$

$\mathbf{Z}_{1-\alpha / 2}^{2}=$ standard normal confidence level of $95 \%=\mathbf{1 . 9 6}$

\section{Inclusion criteria}

Postnatal mothers who delivered from St. Francis Hospital Nsambya and consented for the study were included.

\section{Exclusion criteria}

Postnatal mothers who did consent for study or mothers below 18 years were excluded from the study 


\section{Study tools and techniques}

During the study, the following tools were used in data collection;

I. MEOWS chart: This was adopted from the seventh Confidential Enquiry into Maternal and Child Health (CEMACH) report 2003-2005 (Lewis, 2007).

II. The study questionnaire: This was a semi-structured questionnaire in which data from the MEOWS chart and clinical notes was entered.

Pregnant mothers were recruited in the study by the Research Assistant (RA) in the labour suite when they come for delivery and observation on MEOWS started immediately after delivery. Research assistants had the respondents briefed concerning their roles and expectations during the research.

Data was collected using an interviewer questionnaire with open and closed ended questions from between January and February 2016 by trained Research Assistant and Principal Investigators. Data was extracted from the clinical notes during the follow up by the Principal Investigators for any evidence of obstetrics morbidity. All data were then concentrated to the Principle Investigator who later verified entries made in the data collection tools to confirm correctness of entries into the corresponding fields and to enable processing of data. 
DOI: 10.21522/TIJCR.2014.05.01.Art006

ISSN: $2520-3096$

\section{OBSTETRIC EARLY WARNING CHART}

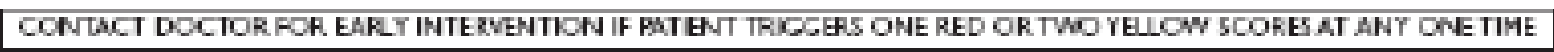
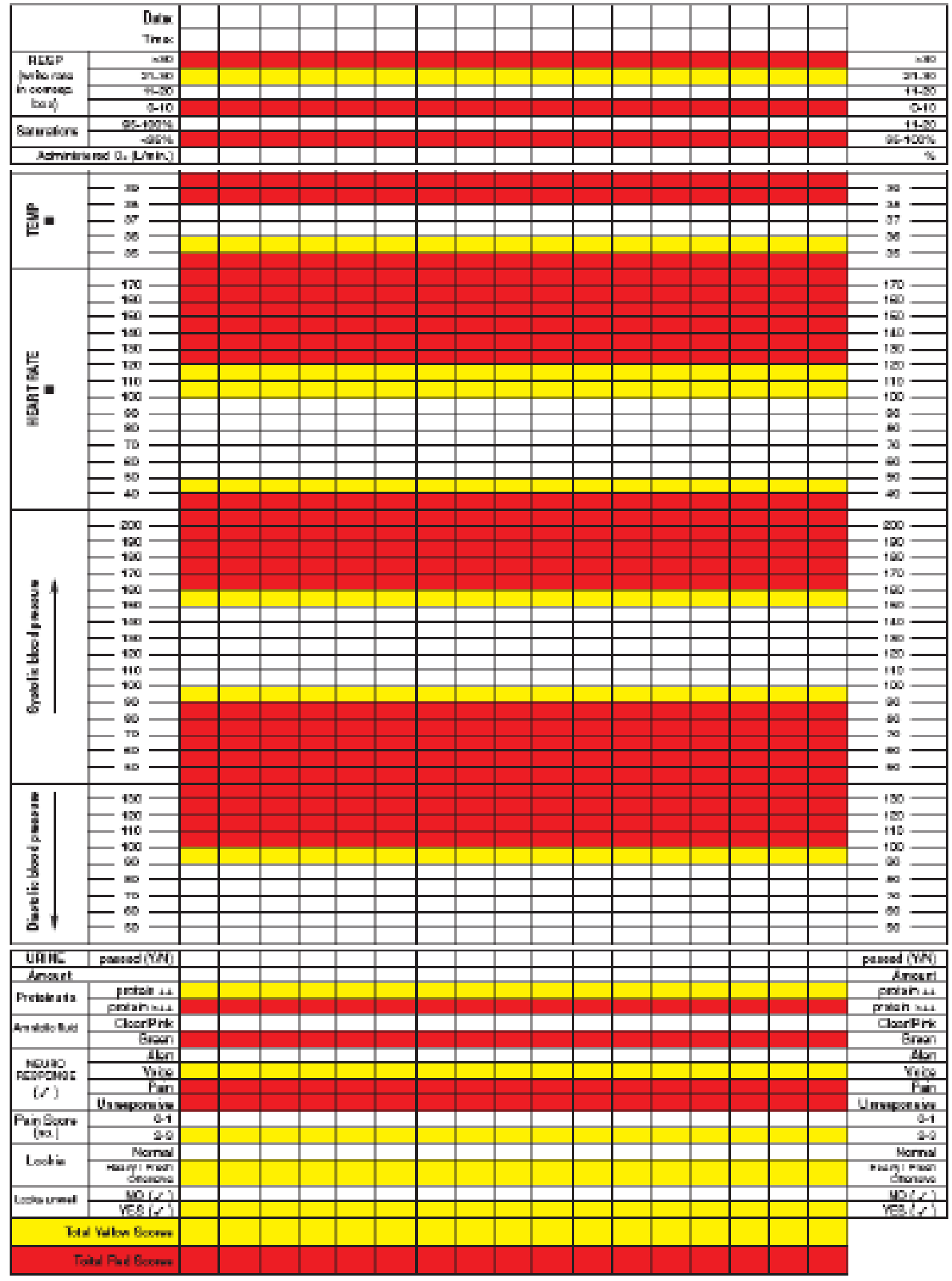

Figure 2. Copy of MEOWS Chart 


\section{Ethical procedures and considerations}

The following ethical procedures were undertaken;

I. Consultation with senior supervisors, other medical personnel and eventual testing of the MEOWS tool was done.

II. Training four midwives and two senior house officers as research assistants was done. They were trained on how to use the MEOWS chart and initiate a call out algorithm.

III. Pre-testing the MEOWS chart on postnatal mothers for one week until efficiency was $100 \%$ on procedural and responsibility arrangements by the research team was carried out. The Midwives and Doctors were made aware of the importance of accurate charting of the patients' parameters, the need for mandatory callout and ensuring of the medical review by Doctors.

\section{Study procedures and techniques}

During the study period, the postnatal mothers were monitored immediately after delivery with MEOWS chart every two hours in the first 24 hours by midwives.

When postnatal mothers were triggered ( 1 red or 2 yellow), a Doctor was called urgently to review the patients on bedside and those who had no trigger were review at 6 hours post natal.

Measurement of temperature (axillary) was done with digital thermometer, blood pressure taken with mercury Blood Pressure Machine (Dekamet Accoson sphygmomanometer made in England). Respiratory rate was taken by counting and timing with clock for one minute. Heart rate and oxygen saturation was taken with pulse-oxymeter. Consciousness level of mothers (AVPU: A: Alert of time, place \& person, score white colour _ Normal, V: response to voice; score yellow _ moderate abnormal, P: response to pain \& U: unresponsive both score red indicative of very abnormal) was taken.

Pain scores $(0=$ no pain at rest or on movement, $1=$ no pain at rest, slight pain on movement, $2=$ intermittent pain at rest, moderate pain on movement, $3=$ severe pain at rest, severe pain on movement) was done. Pain score of 0-1 was coloured white and considered normal whereas score 2-3 was yellow and considered abnormal.

Thermometer, Blood pressure machine and pulse oximeter were validated on a daily basis for consistent measurement. Pulse oximeter was validated on daily by measuring four normal healthy adults for their oxygen saturation and pulse rate (considered valid when their Oxygen saturation is $>95-100 \%$ and pulse rate between 60-100 beat/minute (Ahrens, 2006)

Digital thermometer was validated on daily basis to measure the ice melting point of ice park got from the freeze, which was considered valid when it read 0.00-0.01 ${ }^{\circ} \mathrm{C}$ (Mangum et at., 1995).

Mercury sphygmomanometer was validated daily by checking technical features determining the accuracy of mercury sphygmomanometers. The following features were realized; the top of the mercury meniscus rested at exactly zero without pressure applied. To avoid Substantial errors, manometer was kept vertical during measurement and the air vent at the bottom of the manometer and was kept patent to prevent the mercury column from responding sluggishly and from overestimating pressure.

A trigger was defined as a single abnormal observation (red trigger), or the combination of two mildly abnormal observations (two yellows triggers). A trigger initiated urgent medical assessment by the Doctors and no trigger postnatal mothers were reviewed after 6hours for medical assessment and MEOWS score. Outcome at discharge (maternal morbidity, death, intensive care unit admission \& discharged alive) was retrieved from hospital record and notes. The definitions of maternal morbidity were agreed jointly by the principal investigators and my Supervisors at the beginning of the study and the Doctors were the ones to confirm the morbidity.

Check list on MEOWS chart was done at 6 hours and 24 hours by principal investigator / research assistants for completeness. Appendix 3.0 shows the checklist. 
DOI: $10.21522 /$ TIJCR.2014.05.01.Art006

ISSN: $2520-3096$

Table 1. Table showing limit of triggers for MEOWS parameters

\begin{tabular}{|l|l|l|}
\hline Parameters & Red trigger & Yellow trigger \\
\hline Temperature & $<35$ or $>38$ & $35-36$ \\
\hline Systolic BP $; \mathrm{mmHg}$ & $<90$ or $>160$ & $150-160$ \\
\hline Diastolic BP $; \mathrm{mmHg}$ & $>100$ & $90-100$ \\
& & \\
\hline Heart rate; b/m & $<40$ or $>120$ & $100-120$ \\
\hline Respiratory rate $; \mathrm{b} / \mathrm{m}$ & $<10$ or $>30$ & $21-30$ \\
\hline Oxygen saturation $\%$ & $<95 \%$ & - \\
\hline Pain score & - & $2-3$ \\
\hline Neurological response & Unresponsive, pain & voice \\
\hline
\end{tabular}

\section{Variables}

The following were the primary outcome variables; postpartum haemorrhage, preeclampsia, suspected infection, pulmonary emboli, pulmonary oedema, ruptured uterus, emergency hysterectomy, anaesthetic complications, obstetric injury, prolong hospital stay, admission to intensive care unit and maternal death.

\section{Data management, analysis and presentation}

Questionnaires were cross checked at the end of each day to ensure correctness and completeness. Data was coded and double entered into EPI data version 3.1, cleaned and exported to SPSS version 16.0 for statistical analysis.

Sensitivity, specificity, positives predictive value and negative predictive value were calculated.

The validity of MEOWS was calculated as follows as shown in table 2 below;

Table 2. Calculation of sensitivity, specificity and predictive values

\begin{tabular}{|l|l|}
\hline Trigger had morbidity & Trigger had no morbidity \\
\hline True Positive (TP) & False Positive (FP) \\
\hline No trigger had morbidity & $\begin{array}{l}\text { No trigger had no } \\
\text { morbidity }\end{array}$ \\
\hline False Negative (FN) & True Negative (TN) \\
\hline
\end{tabular}

1. Sensitivity $=\left[\frac{T P}{\left(T P_{P+N N}\right)}\right] \times 100 \%$

2. Specificity $=\left[\frac{(T P+N F N)}{(T N+F P)}\right] \times \underset{T P}{100 \%}$

3. Positive predictive $=\left\{\frac{T P}{(T P+F P)}\right] \times 100 \%$

4. $\quad$ Negative predictive value $=\left[\frac{T N}{(T N+F N)}\right] \times 100 \%$

\section{Quality control}

This was enhanced by training of the 4 Midwives and 2 Senior House Officers as research Assistant and rehearsal of tasks. These were Midwives and Senior House Officers employed in St. Francis Hospital Nsambya who was not on duty. Weekly meetings between Research Assistants and Principal investigators were held to review experiences, performance or progress. The questionnaires were translated into Luganda and back into English: both were used as necessary. Spot checks were done, editing in the field and at the end of the day and reviewing the questionnaires for completeness were done.

\section{Results}

\section{Introduction}

A total of 517 postnatal mothers were recruited in the study and monitored with MEOWS chart by midwives every 2 hour in first 24hours. Of these, 502 were analysed (97\%). Of the 502 mothers, 160 triggered (1 red or 2 yellow) accounting for $31.9 \%$ and they had urgent medical evaluation by the doctor 
whereas of 342mothers didn't, "no triggered" and they had medical evaluation done at 6 hours postnatal by doctors.

Both categories had their MEOWS chart reviewed and clinical note at discharged for evidence of obstetric morbidity. The results were as follows; Triggered had morbidity was 58 (TP), Triggered had no morbidity was 102 (FP), No triggered had morbidity was 13 (FN) and No triggered had no morbidity 329 (TN). See figure 2 below;

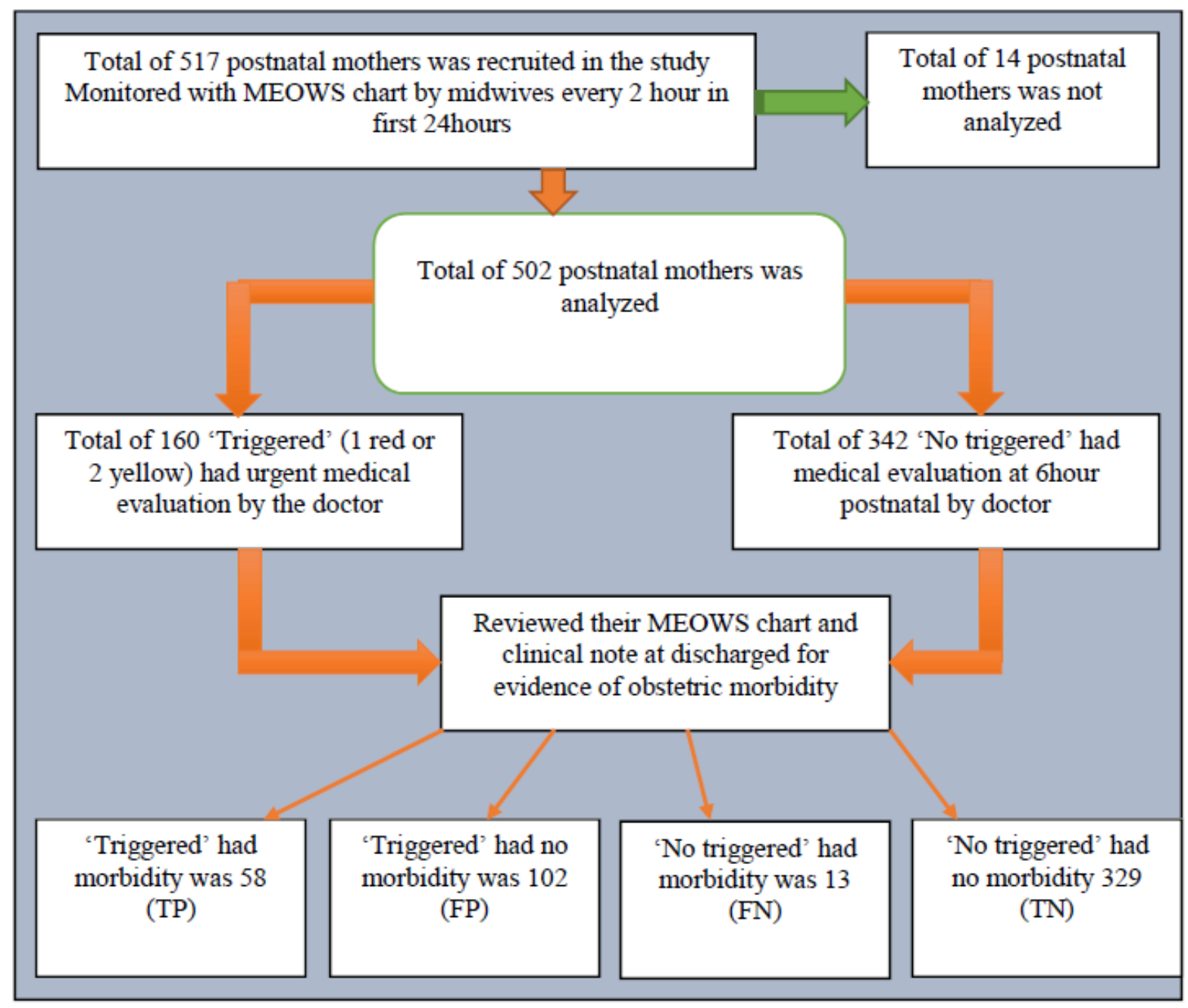

Figure 2. Flow chart of result summary

TP $=$ True Positive, FP = False Positive, FN = False Negative and TN = True Negative

\section{Results on demographic characteristics}

Of the study participants, $59 \%$ of the respondents were of the age between 26 to 35 years with mean age of 28.2 years. Educationally; primary level was at $5.0 \%$ Secondary levels was at $52.1 \%$ and Tertiary levels was $41.7 \%$. Tribe distribution of the postnatal respondents; $59.4 \%$ of the respondents were from the central region of Uganda and other regions accounted 37.0\% whereas none Ugandans were 3.6\%

The results on demographic characteristics were as shown in table 3 below; 
DOI: $10.21522 /$ TIJCR.2014.05.01.Art006

ISSN: $2520-3096$

Table 3. The demographic characteristic of participant

\begin{tabular}{|l|l|l|}
\hline Characteristic & $\begin{array}{l}\text { Number of postnatal } \\
\text { mothers monitored } \\
\text { with MEOWS }\end{array}$ & Percentage \\
\hline Age (years) & & \\
\hline$<25$ & 159 & $31.6 \%$ \\
\hline $\mathbf{2 6 - 3 5}$ & 296 & $59 \%$ \\
\hline$>\mathbf{3 6}$ & 47 & $9.4 \%$ \\
\hline Tribes & & \\
\hline Central region & 299 & $59.4 \%$ \\
\hline Other region & 186 & $37 \%$ \\
\hline None Ugandan & 18 & $3.6 \%$ \\
\hline Religion & & \\
\hline Protestant & 114 & $22.7 \%$ \\
\hline Catholic & 232 & $46.1 \%$ \\
\hline Muslim & 62 & $12.3 \%$ \\
\hline Born again & 79 & $15.7 \%$ \\
\hline SDA & 9 & $1.8 \%$ \\
\hline $\begin{array}{l}\text { Level of } \\
\text { Education }\end{array}$ & & \\
\hline None & 5 & $1.0 \%$ \\
\hline Primary & 25 & $5.0 \%$ \\
\hline Secondary & 262 & $52.1 \%$ \\
\hline Tertiary & 210 & $41.7 \%$ \\
\hline
\end{tabular}

\section{Results on obstetric history of respondents}

Of the study participants; antenatal care attendant was at 99\% (497), HIV positive were at 5.4\% (27). Mode of delivery was as follows; 56.4\% (283) had vaginal delivery, 43\% (216) had caesarean section and $0.6 \%$ (3) instrumental delivery (vacuum- extraction).

The results on obstetric history of mothers were as follows;

Table 4. Obstetric history of respondents

\begin{tabular}{|l|l|l|}
\hline Characteristic & $\begin{array}{l}\text { Number of postnatal } \\
\text { mothers monitored with } \\
\text { MEOWS }\end{array}$ & Percentage \\
\hline Antenatal care & & \\
\hline Attended & 497 & $99.0 \%$ \\
\hline Not attended & 5 & $1.0 \%$ \\
\hline HIV status & & \\
\hline Positive & 27 & $5.4 \%$ \\
\hline Negative & 466 & $92.8 \%$ \\
\hline Unknown & 9 & $1.8 \%$ \\
\hline Parity & & \\
\hline $1-3$ & 369 & $73.5 \%$ \\
\hline 4 and above & 133 & $26.5 \%$ \\
\hline Mode of delivery & & $56.5 \%$ \\
\hline Vaginal & 283 & $42.9 \%$ \\
\hline Caesarean section & 216 & $0.6 \%$ \\
\hline Instrumental delivery & 3 & \\
\hline & & \\
\hline
\end{tabular}




\section{Results based on morbidity factors of mothers}

One hundred and sixty patients (31.9\%) of the mothers triggered and of which; 11.5\%(58patients) had obstetric morbidity, including postpartum haemorrhage $35.5 \%(21)$, preeclampsia $26.3 \%$ (15), suspected infection $22.4 \%$ (13), third degree perineum tear 5.3\% (3), anaesthetic complications $4 \%$ (2) and prolong hospital stay $7 \%$ (4).

\section{Results on sensitivity, specificity and predictive values}

The Results on sensitivity, specificity and predictive values are as below;

Table 5. Calculation of sensitivity, specificity, positive predictive value and negative predictive values

\begin{tabular}{|l|l|}
\hline Trigger has morbidity & Trigger has no morbidity \\
\hline True Positive $(\mathrm{TP})=58$ & False Positive $(\mathrm{FP})=102$ \\
\hline No trigger has morbidity & No trigger has no morbidity \\
\hline False Negative $(\mathrm{FN})=13$ & True Negative $(\mathrm{TN})=330$ \\
\hline
\end{tabular}

Sensitivity $=\left[\frac{T P}{(T P+F N)}\right] \times 100 \%=\left[\frac{58}{(58+13)}\right] \times 100 \%=81.7 \%$

Specificity $=\left[\frac{T N}{(T N+F P)}\right] \times 100 \%=\left[\frac{329}{(329+102)}\right] \times 100 \%=76.3 \%$

Positive predictive value $=\left[\frac{T P}{(T P+F P)}\right] \times 100 \%=\left[\frac{58}{(58+102)}\right] \times 100=36.3 \%$

Negative predictive value $=\left[\frac{T N}{(T N+F N)}\right] \times 100 \%=\left[\frac{329}{(329+13)}\right] \times 100 \%=96.2 \%$

\section{Summary of findings}

The results for this study versus the acceptable normal threshold ranges (in brackets) are as follow;

1. Sensitivity of MEOWS in predicting maternal morbidity was $81.7 \%$ (95\% Confident Interval; $80 \%-95 \%)$

2. Specificity $76.3 \%$ (95\% Confident Interval; $74-82 \%$ )

3. Positive predictive value $36.3 \%$ (95\% Confident Interval; $30-44 \%$ )

4. Negative predictive value $96.2 \%$ (95\% Confident Interval; 94-99\%).

\section{Discussion, conclusion and recommendation}

\section{Discussion}

The values of MEOWS in this study is consistent with other findings; Sensitivity $81.7 \%$ (95\% Confident Interval; 80\%-95\%), Specificity 76.3\% (95\% Confident Interval; 74-82\%), Positive predictive value $36.3 \%$ (95\% Confident Interval; 30-44\%) and Negative predictive value $96.2 \%$ (95\% Confident Interval; 94-99\%) as the results of Singh, McGlennan, England and Simons (2012)

In this study the specificity was $76.3 \%$ and is comparable to $79 \%$ done in UK by Singh, McGlennan, England and Simons (2012).

\section{Conclusions}

MEOWS chart can be used as a monitoring tool for predicting maternal morbidity, safe mother life and reduce the burden of severe morbidity and maternal mortality.

\section{Recommendation}

MEOWS chart should be adapted for use as monitoring tool for inpatients in maternity ward in low resource settings like Uganda. 
DOI: 10.21522/TIJCR.2014.05.01.Art006

ISSN: 2520-3096

\section{References}

[1].Ahrens, T. S. O. K. (2006). Comparison of Three New Generation Pulse Oximeters in a Medical Intensive Care Unit. Crit Care Med. 2006, 34: A85.

[2].Buderer, N. M. (1996). Statistical methodology: Incorporating the prevalence of disease into sample size calculation for sensitivity and specificity. Acad Emerg Med 1996, 3:895-900.

[3].Cioffi, J. (2000a) Nurses' experiences of making decisions to call emergency assistance to their patients. Journal of Advanced Nursing, 32: 108-114.

[4].ICF International Inc. (2012). Uganda Demographic and Health Survey 2011. Kampala: UBOS.

[5].Isaacs, R. A., Wee, M. Y. K., Bick, D. E., Sheppard, Z. A., Thomas, S., Hundley, V., Smith, G. B., Van.

[6].Teijlingen, E. and Thomas, P. W. (2014). A national survey of obstetric early warning systems in the United Kingdom: five years on. Journal of the Association of the Anaesthetists of Great Britain and Ireland, 69 (7). https://doi.org/10.1111/anae.12708.

[7].Lewis, G. (ed). (2007). the confidential Enquiry into Maternal and Child Health (CEMACH) Saving Mothers' Lives: reviewing maternal deaths to make motherhood safer - 2003- 2005. The Seventh Report on Confidential Enquiries into Maternal Deaths in the United Kingdom. London: CEMACH.

[8].Mangum, B.W. (1995). "Reproducibility of the Temperature of the Ice Point in Routine Measurements," NIST Tech. Note 1411.

[9].Marc, V. D. V., Helen, S. and Lauren, A. P. (2013). Maternal Critical Care: A Multidisciplinary Approach. New York: Cambridge University Press.

[10]. Melanie, F. C. (2014). A modified early obstetric warning system. British Journal of Midwifery, 22 (12).

[11]. Ministry of Health [MOH]. (2010). Statistical Abstract 2010. Kampala: Author Say, L., Chou, D., Gemmill, A., Tunçalp Ö., Moller, A. B., Daniels, J. D. (2014). Global Causes of Maternal Death: A WHO Systematic Analysis. Lancet Global Health, 2 (6): 323-333.

[12]. Singh, S., McGlennan, A., England, A. and Simons, R. (2012). A validation study of the CEMACH recommended modified early obstetric warning system (MEOWS). Anaesthesia, 67(1), 12-18. doi:10.1111/j.1365-2044.2011.06896.x.

[13]. Uganda Bureau of Statistic [UBOS]. (2012). Uganda Demographic and Health Survey 2011. Kampala: Author.

[14]. United Nations [UN]. (2015). Global Strategy for Women's, Children's and Adolescents' Health, 2016-2030. New York: Author.

[15]. World Health Organization [WHO]. (2005). World Health Report. Geneva: Author. 